Trauma Berufskrankh 2012 · 14:3-4 DOI 10.1007/s10039-011-1829-6 Online publiziert: 15. Februar 2012

(c) Springer-Verlag 2012

\author{
U.-J. Gerlach \\ Septische Knochen- und Gelenkchirurgie, BG-Unfallkrankenhaus Hamburg
}

\title{
Septische Komplikationen in der Orthopädie und Unfallchirurgie
}

Septische Komplikationen (Knochenund Gelenkinfektion) sind schwerwiegende Komplikationen der Orthopädie und Unfallchirurgie. Ihre Behandlung ist oft aufwendig, langwierig und kostenintensiv. Das Heilverfahren wird deutlich verlängert, und die Kosten steigen erheblich. Nicht selten kommt es zu höheren Rentenzahlungen. Für den Versicherten resultiert aufgrund des langen Heilverfahrens die soziale und berufliche Isolierung.

Die Behandlung von posttraumatischen und postoperativen Knochen- und Gelenkinfektionen ist häufig multidisziplinär und bedarf spezieller Kenntnisse. Je frühzeitiger die septische Komplikation diagnostiziert oder wahrgenommen und je früher die konsequente radikale Therapie begonnen wird, desto günstiger ist der Verlauf. Bei von septischen Komplikationen Betroffenen handelt es sich häufig um multimorbide Patienten. Nicht selten finden sich problematische Weichteilverhältnisse nach vorausgegangenen mehrfachen Operationen. Das lange Heilverfahren bedingt oft Gelenkkontrakturen und Bewegungsmangelschäden. Umso entscheidender ist es, frühzeitig die Diagnose zu stellen und die Behandlung mit einem klaren therapeutischen Konzept durchzuführen. Sie erfolgt in einer großen Zahl der Fälle in den septischen Abteilungen der BGKliniken (BG: Berufsgenossenschaft).

Basis für eine korrekte Diagnostik sind eine ausführliche Anamneseerhebung und eine körperliche Untersuchung. $\mathrm{Zu}$ den diagnostischen $\mathrm{Ma} ß n$ nhmen gehören die Projektionsradiographie, die Computertomographie (CT) und die Magnetre- sonanztomographie (MRT). In neuer Zeit wurde das Spektrum durch die PET-CT (PET: Positronenemissionstomographie), ein bildgebendes Verfahren mit hoher Treffsicherheit, erweitert. Moderne molekularbiologische diagnostische Maßnahmen befinden sich noch im Stadium der Grundlagenforschung, zu nennen ist hier das ,protein profiling " in Gewebe und Serum für eine individuelle Diagnostik sowie auch das Therapiemonitoring bei Osteomyelitis.

Die Behandlung der septischen Komplikation in der Orthopädie und Unfallchirurgie sollte auf einem auf den Patienten abgestimmten individuellen Konzept beruhen.

Die Behandlung der Knocheninfektion muss mehrzeitig durchgeführt werden. Im ersten operativen Schritt ist ein radikales Débridement der infizierten Knochen und Weichteile erforderlich. Im zweiten Schritt sollten möglicherweise vorliegende Haut-/Weichteildefekte zeitnah stabil gedeckt werden, entweder durch lokale Maßnahmen oder durch frei transplantierte Lappen. Hierbei bewährte sich die Zusammenarbeit mit den Plastischen Chirurgen. Im dritten operativen Schritt, nach Infektberuhigung, steht der Knochendefektaufbau im Vordergrund, entweder durch Spongiosaplastik oder durch Segmenttransport.

Im Falle von Gelenkinfektionen ist eine radikale Synovektomie indiziert. Bei akuten Gelenkinfektionen (innerhalb der 1. Woche) handelt sich um einen unfallchirurgischen Notfall. Chronische oder arthroskopisch vorbehandelte Gelenkin- 
fektionen sollten offen, d. h. durch Arthrotomie, behandelt werden.

Das Vorgehen bei periprothetischen Infektionen hängt von der Dauer der aufgetretenen Symptome ab. Innerhalb der ersten 4 Wochen ist ein Erhaltungsversuch mit Wechsel aller austauschbaren Teile legitim, nach 4 Wochen hat ein derartiges Vorgehen keine Erfolgsaussichten. Es muss dann die Endoprothese explantiert werden, die Reimplantation kann ein- oder zweizeitig durchgeführt werden.

Die Entwicklung hinsichtlich Diagnostik und Therapie wandelt sich. Hier halten zunehmend molekularbiologische Maßnahmen Einzug, die gleichzeitig eine Risikoabschätzung im Verlauf erlauben. Vermehrt finden mesenchymale Stammzellkonzentrate als Zugabe zur Spongiosa Verwendung. Sie dienen der Verbesserung des Einbaus oder werden beim Segmenttransport zum schnelleren knöchernen Remodelling in die Transportstrecke injiziert. Additive Maßnahmen wie die Behandlung mit Exogenultraschall oder die Injektion von Parathormon komplettieren die therapeutischen Möglichkeiten. Gerade in den BG-Klinken wird intensiv an der Weiterentwicklung der bisherigen Behandlungskonzepte geforscht. Unterstützt werden Forschungsprojekte durch das Bundesministerium für Bildung und Forschung (BMBF).

Im vorliegenden Schwerpunktheft werden in Übersichtsartikeln neue Entwicklungen hinsichtlich Diagnostik, Therapie und Risikoabschätzung dargestellt. Gleichzeitig wird die Problematik von multiresistenten Erregern bei Gelenkinfektionen aufgezeigt.

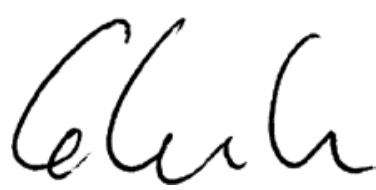

\section{Dr. Ulf-Joachim Gerlach}

\section{Korrespondenzadresse}

\section{Dr. U.-J. Gerlach}

Septische Knochen- und Gelenkchirurgie, BG-Unfallkrankenhaus Hamburg, Bergedorfer Straße 10, 21033 Hamburg u.j.gerlach@buk-hamburg.de

\section{EFORT 2012}

\section{Die Europäische Orthopädie und Unfallchirurgie kommt nach Deutschland}

Der EFORT Kongress ist der größte internationale Kongress für Orthopäden und Unfallchirurgen in Europa und zieht mittlerweile Teilnehmer aus über 90 Ländern an. Zum zweiten Mal nach 1995 wird nun im nächsten Jahr der Jahreskongress in Deutschland stattfinden: vom 23. bis 25. Mai 2012 lädt EFORT nach Berlin ein und die Teilnehmer erwartet ein hochkarätiges wissenschaftliches Programm. Es sind mehr als 3.900 Abstracts eingereicht worden, eine neue Rekordzahl in der Geschichte der europäischen Gesellschaft.

Aufgrund der UN Dekade für Verkehrssicherheit wird ein Schwerpunktthema die Behandlung von Verletzungen und Verletzungsfolgen sein, ein zweites aufgrund des European Year for Active Aging 2012 die Behandlung der degenerativen Erkrankungen der Haltungs- und Bewegungsorgane.

Dieser Kongress erlaubt in einer einzigartigen Art und Weise die gesamte Breite des Faches zu erfahren, gewürzt durch unterschiedliche länderspezifische Ansichten und internationale Experten der Spitzenklasse.

Beliebte und bewährte Formate wie Symposia und Instructional Lectures (IL) werden durch interaktive Formate wie z.B. Controversial Case Discussions (CCD) und Expert Meet Experts (ExMEx) Sessions ergänzt. Neue Formate, wie etwa Crossfire Sessions, bei denen zwei gegensätzliche Ansätze zur Problemlösung vorgestellt und diskutiert werden, erlauben kontroverse Diskussionen über unterschiedliche Philosophien mit Meinungen aus verschiedenen Ländern. EFORT's Specialty Societies haben Sitzungen zu verschiedenen Schwerpunktthemen vorbereitet, in denen ausgewiesene Experten ihre Erfahrung mitteilen.

Der eintägige Comprehensive Review Course (CRC) richtet sich vorwiegend an jüngere Kollegen und erlaubt einen kompetenten Überblick über das gesamte Fach an einem Tag.

Wir freuen uns darauf mit Ihnen die internationalen Gäste in der deutschen Hauptstadt begrüßen zu dürfen, die für viele ausländische Kollegen eine besondere Anziehungskraft hat. Wir sind überzeugt, dass es auch für die deutschen Orthopäden und Unfallchirurgen eine einmalige Möglichkeit ist, sich in diesem Rah- men fortzubilden und zudem mit internationalen Ansichten auseinandersetzen zu können. Als EFORTs Kongresspräsidenten und Organisationsteam freuen wir uns, Sie im Mai 2012 in Berlin begrüssen zu dürfen. Sie finden alle weiteren Informationen auf http://www.efort.org/ berlin2012/.

Prof. Dieter C. Wirtz und Prof. Karsten E. Dreinhöfer mit Florian Gebhardt, Ulrich Stöckle, Karl-Dieter Heller und Klaus-Peter Günther 